ITP-UU-02/68

SPIN-2002/44

hep-th/yymmnnn

\title{
THE HIDDEN INFORMATION IN THE STANDARD MODEL*
}

\author{
Gerard 't Hooft \\ Institute for Theoretical Physics \\ Utrecht University, Leuvenlaan 4 \\ 3584 CC Utrecht, the Netherlands \\ and \\ Spinoza Institute \\ Postbox 80.195 \\ 3508 TD Utrecht, the Netherlands \\ e-mail: g.thooft@phys.uu.nl \\ internet: http://www.phys.uu.nl/ thooft/
}

\section{Astract}

July 21, 2004

*Presented at the International Symposium on Frontiers of Science, in honor of C.N. Yang, Tsinghua University, Beijing, June 18, 2002 


\section{Introduction}

Many of the advances in twentieth century theoretical physics can be attributed to the discovery and subsequent exploitation of symmetries. Not only Special and General Relativity embody the importance of realizing how a precise analysis of Nature's symmetries can enhance our understanding, but also Quantum mechanics itself owes much of its success to the exploitation, to the extreme, of symmetries such as rotation and translation symmetries, but also conformal symmetries and many kinds of internal and accidental symmetries. It was Frank Yang's deep insights that allowed him to observe how to generalize the few local symmetry concepts that existed at his time: local coordinate transformations in general relativity and the Weyl gauge invariance of electromagnetism.

Together with Robert Mills, he showed that one can write down completely selfconsistent field equations for a set of fields which are very similar to electric and magnetic fields, but which induce transitions of particles that pass through such fields into other, related species of particles.[1] The group of local gauge transformations, which was an Abelian group in the electro-magnetic case, is now replaced by a non-Abelian group, which may directly be linked to groups such as that of the isospin transformations, which up to that time had been treated only as global symmetry groups.

The theory naturally requires many particle species to form representations of such global groups, and the fact that global, non-Abelian symmetry groups already seemed to be applicable to describe the known particle species - first $S U(2)$ and then, later, $S U(3)$, could be seen as a strong indication that these ideas could become quite meaningful and important. In spite of this, however, the Yang-Mills scheme received an amount of skepticism as if it were some fringe science, during the first 15 years. [2]

Even the work of R. Brout and F. Englert, [3] and independently of P. Higgs, [4] did little to alter that status. I think that one can present several explanations for this disinterest. One is, that investigators were still searching for Nature's fundamental principles. Could gauge invariance be a fundamental principle? Then why introduce scalar fields that turn gauge symmetry into something that seems to be much uglier? Why should one rely on Quantum Field Theory, if non-perturbatively this theory appeared to exhibit unacceptable small-distance behaviour, whereas the perturbative formulation appears to suffer from unacceptable infinite renormalization parameters? Presently, we do know what the solid and reliable principles are on which to construct theories: Quantum Field Theories with Yang-Mills fields in them, do possess acceptable small distance features, and gaugeinvariance is an acceptable but not exhaustive starting point for a theory; by adding these gauge fields, theories can be obtained with approximate or even exact asymptotic freedom, but this does not exclude the inclusion of elementary non-gauge fields, which may be scalar or fermionic, or, in the most satisfactory schemes, we may add both. 


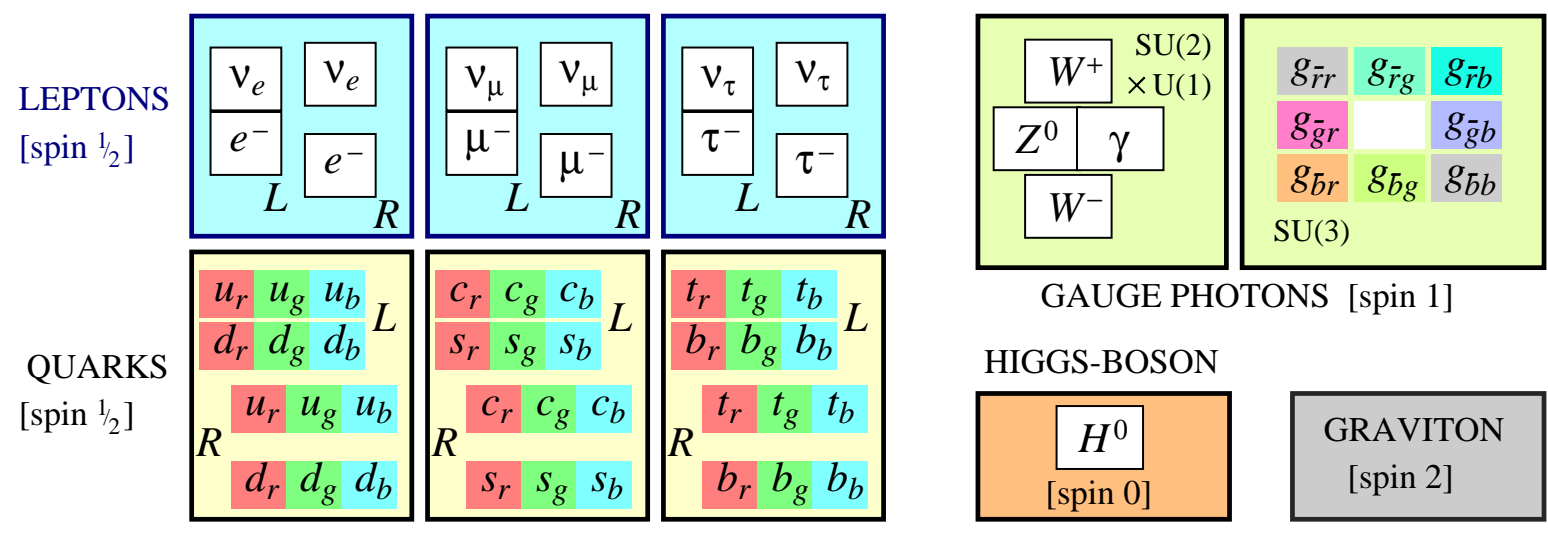

Figure 1: The Standard Model of the Elementary Particles

\section{The Standard Model and beyond.}

These insights lead to the Standard Model, see Fig. 1. It is fully renormalizable at the perturbative level, its infinities are fully under control, in particular because the anomalies cancel out, and it is very nearly asymptotically free, at least to such an extent that no corrections to the model are needed until extremely high energies would be reached, far beyond what is presently attainable in particle accelerators. The rest of this section will be used to admire the beauties of the Standard Model, without yet attempting to say anything new to the experts.

The Standard Model agrees surprisingly well with the experimental observations. Indeed, for more than 20 years, physicists have tried to detect deviations from the Standard Model predictions. What was found, instead, is slight improvements in the details that were known. Most of the objects that originally were still missing have been found - with as yet the only exception being the Higgs boson - , most of the values of the freely adjustable physical constants were determined much more accurately, and some more delicate improvements were made: neutrinos received mass values and mixing angles much like their colored counterparts, the quarks.

The extraordinary successes of the Standard Model allowed us, for the first time, to speculate about Nature'a Laws at a domain of energies and distance scales far beyond what can be reached directly in most experiments. First, one may observe the great similarity between the quark quantum numbers and the lepton quantum numbers. The newly established fact that neutrinos have masses and mixing angles only further enhances this similarity. It is therefore natural to expect that $S U(3)_{\text {color }}$ and $U(1)$ emerge as unbroken subgroups from a spontaneously broken $S U(4)$. The scale at which this spontaneous breaking should occur will probably be extremely high, so as to allow for the strong coupling coefficient to run towards the $U(1)$ coupling strength. In any case, present precision measurements do not betray the presence of heavy vectors with lepto-quark quantum numbers.

Another extension of the Standard Model may be suggested by adding a right-handed 
$S U(2)$ gauge field, turning $S U(2)_{\text {left }} \times U(1)$ into $S U(2)_{\text {left }} \times S U(2)_{\text {right }}$. The algebra of this group coincides with that of $S O(4)$.

The algebra of the $S U(4)$ group of the strong interactions coincides with that of $S O(6)$. The last step is then to join $S O(4)$ and $S O(6)$ into $S O(10)$. The possibility of this last step is so delicate that it is seen as a strong argument in favor of the previous steps towards this construction. The fermions form three generations of a single spinorial $\mathbf{1 6}$ representation of this group. As they are also spinors with respect to ordinary space-time rotations, one may even suspect a Kaluza-Klein compactification process here.

Also at this last step in unification, the strong and the weak coupling strengths must converge. This is why this unification mechanism must happen at a unification scale which is near the Planck scale. However, the left-right symmetry restoration of the previous step requires the $S U(2)$ and the $U(1)$ forces to coincide, which also requires these to run until a similar unification scale is reached. The fact that these various steps of unification all appear to take place at nearly the same energy, close to the Planck scale, is an important piece of information to be drawn from our present understanding of the Standard Model.

The above arguments are of course not new. It is important however to stress that, so far, no supersymmetry or superstring theories were needed for these observations. Supersymmetry does allow us to construct a theory where the left-right symmetry braking and the $S O(10)$ breakdown happen precisely simultaneously. This is often seen as a clue pointing towards a supersymmetric extension of the Standard Model. This signal is perhaps not very strong, but it is also unsatisfactory to attribute it to coincidence.

\section{Questions.}

While staring at these conspicuous and at the same time mysterious structures, we are prompted to ask many questions. Some of these questions have been discussed at numerous occasions by many authors. For instance:

- Why do there appear to be exactly three generations of quarks and leptons?

- Where do these wildly varying mass values come from? There appears to be a hierarchy of scales in Nature, generated by this hierarchy of mass values. Why do we have so many numbers whose orders of magnitude span such a wide range?

- Why in particular these groups and these representations? Is it a coincidence that the spinors also form a spinorial 16 representation of $S O(10)$ ? Why are the Higgs scalars arranged in the representations that we see?

- Is there supersymmetry?

- How do we include the gravitational force? Is it a coincidence that the Planck scale and the unification scale appear to be fairly close together? 
These are the questions that we have been unable to answer, in spite of many vigorous attempts.

In this lecture, I would like to put more emphasis on questions that are not heard as often, while they may be equally important to ask:

$\diamond$ Can gravity (and, in particular, curved, closed universes) be reconciled with the rules of Quantum Mechanics at all? Can the Universe as a whole form states in a Hilbert space, while the concept of an 'observer', who should be sitting outside this universe, would be a notion that is hardly acceptable?

$\diamond$ How important is the notion of locality in physics? Those who investigate the foundations of Quantum Mechanics, and those who try to interpret Super String Theory, are often tempted to drop locality as a necessary feature of the Laws of Physics. But can we make sense at all of a framework describing our world if we allow action-at-a-distance everywhere?

$\diamond$ How can local gauge invariance arise in a deeper fundamental theory?

$\diamond$ How can a naturally small (or vanishing) cosmological constant arise — while fermions and bosons only match approximately, if at all?

\section{Holography}

My motivation for asking these questions requires further clarification. We know that the gravitational force is fundamentally unstable. Unlike the electromagnetic case, like 'charges' attract one another, so that gravity could become an accumulative force. In general relativity, the instability against implosion leads to one stationary solution in which all matter imploded completely: the black hole. There exist some misconceptions concerning black holes. Some authors appear to believe that the description of a black hole requires the assumption that matter forms a singularity first, and that, by assuming possible exotic laws of physics, one might be able to avoid the formation of a black hole altogether. However, the most characteristic feature of a black hole is the emergence of a horizon. The singularity could be affected by exotic laws of Nature, but this is largely immaterial for our understanding of a black hole. The emergence of the horizon is purely a consequence of General Relativity, requiring nothing more than the equivalence between inertial and gravitational mass, in combination with special relativity. Of course, one may question these principles of Nature, but one should realize that they have been corroborated by numerous experiments. The existence of black holes follows from laws of physics that all have been tested to great accuracy.

Applying directly the Laws of Quantum Field Theory to the environment of the horizon, S. Hawking[5] was lead to conclude that particles of all species are emitted, and that they are distributed according to a black body spectrum with an easily computable 
temperature $^{1}$ This beautiful result strongly suggests that black holes are much more mundane forms of matter than the esoteric pure 'balls of gravity' that they appear to be in the classical theory of General Relativity. From their thermal behaviour, one can derive an estimate for the density of states, and from that, one can easily imagine that black holes will blend naturally into the spectrum of ordinary particles at the Planck scale. So, in a sense, black holes and particles are the same things, and, intuitively, we can hardly imagine otherwise.

The expression we obtain for the density of states corresponds to what one would get if all dynamical degrees of freedom of a black hole would be distributed evenly over the horizon. There is one boolean degree of freedom on every $7.24 \cdot 10^{-66} \mathrm{~cm}^{2}$ of the horizon. This number is $4 \ln 2$ times the Planck length squared. It is important to realize that, in a linearized quantum field theory, the number of degrees of freedom would diverge on the horizon. The entropy of a black hole appears to match the degrees of freedom of a quantum field theory only if one performs a hard cut-off at distances comparable to the Planck length from the horizon. [7] At first sight, such a cut-off seems to be forbidden by General Relativity, but one must realize that, at that scale, gravitational forces become strong, so that linearized quantum field theory does not apply; exactly what to do is not properly understood. Applying to the theory with cut-off a general coordinate transformation back to locally regular space-time, one ends up with what is known as the 'holographic principle': there cannot exist more physical degrees of freedom in any closed system than one per $7.24 \cdot 10^{-66} \mathrm{~cm}^{2}$ of its surface area.[8]

It is here that the question of locality comes up: How can one construct a theory which is reasonably consistent with locality features that we perceive in the real world and yet has its physical degrees of freedom distributed evenly over a surface? Can such a theory be consistent with unitarity and causality?

A natural answer to this question may seem to be, that what is needed is a 'topological' theory, a theory where all physical degrees of freedom can be mapped onto the boundary. This is how holography is usually interpreted in string theory. But this does not appear to resolve the problem of locality. How can it be that, nevertheless, we perceive our world as $3+1$ dimensional? From a physical point of view, this seems to be a genuine paradox, not unlike the one that in 1900 lead Max Planck to postulate energy quantization. This time, however, one might be forced to arrive the opposite conclusion: the paradox came from our presumption that states form a quantum mechanical Hilbert space. There must be something wrong with that.[9]

At this point, I think it is totally legitimate to ask: "Why should these issues not be related to the question of the foundation of quantum mechanics?"

And so, one is lead back to the issue of (local?) hidden variables. It is an issue not to be forgotten.

\footnotetext{
${ }^{1}$ Although the value of this temperature is agreed upon by most authors, one may suspect that it hinges on an assumption that is not yet proven to be correct. The author still maintains that an alternative scenario cannot be excluded[6]
} 


\section{Determinism}

Rather than trying to devise tricky 'Gedanken experiments' in which one could search for deterministic features, we start from the other end. Consider some simple deterministic system, consisting of a set of $N$ states,

$$
\{(0),(1), \cdots,(N-1)\}
$$

on a circle. Time is discrete, the unit time steps having length $\tau$ (the continuum limit is left for later). The evolution law is:

$$
t \rightarrow t+\tau \quad: \quad(\nu) \rightarrow(\nu+1 \bmod N)
$$

Note, that this model is representative for anything that shows periodic behaviour in time.

Introducing a basis for a Hilbert space spanned by the states $(\nu)$, the evolution operator can be written as

$$
U(\Delta t=\tau)=e^{-i H \tau}=\left(\begin{array}{ccccc}
0 & & & & 1 \\
1 & 0 & & & \\
& 1 & 0 & & \\
& & \ddots & \ddots & \\
& & & 1 & 0
\end{array}\right) .
$$

The eigenstates of $U$ are denoted as

$$
|n\rangle=\frac{1}{\sqrt{N}} \sum_{\nu=1}^{N} e^{\frac{2 \pi i n \nu}{N}}(\nu), \quad n=0, \cdots, N-1 .
$$

This evolution law can be represented by a Hamiltonian using the notation of quantum physics: ${ }^{2}$

$$
H|n\rangle=\frac{2 \pi}{N \tau} n|n\rangle
$$

This Hamiltonian can be used to describe a quantum harmonic oscillator. It is our proposal to identify the states (5.4) with the eigenstates of an harmonic oscillator. They evolve exactly as required. There is a slight problem, however: the number $n$ is limited to be less than $N$, the number of discrete positions on the circle.

The remedy to this problem, at first sight, appears to be easy: we take the $N \rightarrow \infty$ limit. Indeed, then we get exactly all states of the harmonic oscillator.[10] However, upon further inspection, there still remains an important obstacle. If one wants to introduce interactions, that is, non-harmonic terms in the oscillator, expressions are needed that

relate the 'ontological states' $(\nu)$ to the harmonic oscillator states. One then discovers that an operator that links the ground state $|\nu=0\rangle$ to the highest energy state $|\nu=N-1\rangle$ 


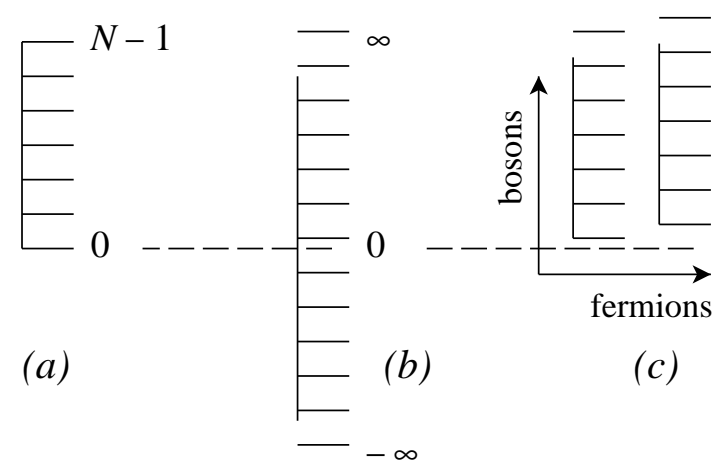

Figure 2: The spectrum of the Hamiltonian for a deterministic, periodic system. a) $N$ points on a circle, rotating with one fixed angular velocity. b) In the continuum limit, it is hard to ignore the negative energy solutions. c) After a sign flip of the negative energy states, we get a double spectrum, suggesting a fermionic degree of freedom.

(see Fig. 2a) continues to contribute to these relations in an essential way. Therefore, the continuum limit is not as smooth as what would be desired.

What we have presently under investigation is the question whether there exists a superior way to obtain a continuum limit. In the continuum limit, one obtains Fig. $2 b$. If the evolution can run along the circle in both directions, the eigenvalues of the Hamiltonian will be plus or minus Eq. (5.5). We may be tempted to turn Fig. $2 b$ into Fig. $2 c$. Since there are then two classes of states, it appears that a fermionic degree of freedom arises. It is too early to conclude from this that supersymmetry has anything to do with a deterministic interpretation, but it could be that fermions will have a natural role to play in our deterministic models. Generally speaking, one finds that fermions are associated to discrete degrees of freedom in a deterministic underlying model. Unfortunately, there are still several problems with this picture.

Since it is easy to imagine that deterministic, periodic systems may influence one another, the inclusion of interactions should not give problems in principle. It seems, however, that the mathematical methods that are familiar to us do not apply. This holds in particular when one tries to incorporate information loss.

\section{Information loss}

The reasons why information loss may be an essential ingredient in deterministic hidden variable models of the sort pioneered above, has been extensively discussed in Ref.[9]. A prototype microcosmos with information loss is the model of Fig. 3. Following the arrows,

\footnotetext{
${ }^{2}$ If so desired, one may replace $n$ by $n+\frac{1}{2}$, by adding a phase factor to the evolution operator $U$ of Eq. (5.3).
} 


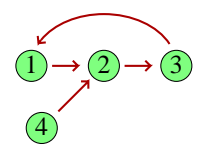

Figure 3: Mini-universe with information loss. The arrows show the evolution law.

one would conclude that the evolution matrix is

$$
U=\left(\begin{array}{llll}
0 & 0 & 1 & 0 \\
1 & 0 & 0 & 1 \\
0 & 1 & 0 & 0 \\
0 & 0 & 0 & 0
\end{array}\right)
$$

This, of course, is not a unitary matrix. One way to restore unitarity would be to remove state \# 4. The problem with that is that, in universes with tremendously many allowed states, it would be very difficult to determine which of the states are like state number 4 , that is, they have no state at all in their (distant) past.

A preferred way to proceed is therefore to introduce equivalence classes of states. Two states are equivalent iff, some time in the near future, they evolve into one and the same state ${ }^{3}$. In Fig. 3, states \#\# 1 and 4 are equivalent, so they form one class. By construction then, equivalence classes evolve uniquely into equivalence classes. In this respect, this universe is like the one described by Eq. (5.2) and the matrix $U$ of Eq. (5.3), with $N=3$.

It should be emphasized that, at the Planck scale, information loss is not a small effect but a very large effect. Large numbers of 'ontological' states are in the same equivalence class, and the equivalence classes form a much smaller set than the class of all states. This is how it can happen that the total number of distinguishable quantum states (= the number of equivalence classes) may only grow exponentially with the surface of a system, whereas the total number of ontological states may rise exponentially with the volume. This seems to be demanded by black hole physics, when we confront the laws of quantum mechanics with those of black holes.

Information loss at the level of the underlying deterministic theory, may also explain the apparent lack of causality in the usual attempts to understand quantum mechanics in terms of hidden variables. The definition of an equivalence class refers to the future evolution of a system, and therefore it should not be surprising that in many hidden variable models, causality seems to be violated. One has to check how a system will evolve, which requires advance knowledge of the future.

Information loss at the Planck scale may also shed further light on the origin of gauge theories. it could be that, at the level of the ontological degrees of freedom at the Planck scale, there is no local gauge symmetry at all, but in order to describe a physical state, that is, an equivalence class, we need to describe a particular member of this class, a

\footnotetext{
${ }^{3}$ It could also happen that two states merge into the same state in the distant future, but in many models merging may become increasingly unlikely as time goes on.
} 
single state. Its relation to the other members of the same equivalence classes could be what is presently called a "gauge transformation".

\section{Final remarks.}

The fact that we need gauge-noninvariant vector potentials and other fields, to describe our world, is a peculiar fact of life that we learned from studying particle physics, not in the least due to the work of C.N. Yang. This, together with other very specific peculiarities of the Standard Model, is what was meant with the words 'hidden information' in the title, although the title may also be regarded as referring to the apparent need for phenomena such as information loss in our basic theories.

An important remark about symmetries:[11] most of the symmetry groups of Nature refer to symmetries in Hilbert Space. In our pre-quantum theories, there will, presumably, be much less symmetry. We expect that the physical degrees of freedom become discrete at the Planck scale, and this implies that, at best, translation symmetry becomes discrete, whereas rotation symmetry may disappear altogether. If an ontological theory can be constructed (a theory describing a single reality), it is likely to require a new form of mathematics allowing us to introduce symmetries relating ontological operators (which we call 'beables') to quantum operators (operators that replace an ontological state by an other state, called changeables). it is these symmetries that then will lead to the symmetries we know from particle physics.

\section{References}

[1] C.N. Yang and R.L. Mills, Phys. Rev. 96 (1954) 191.

[2] H.B. Newman and Th. Ypsilantis, History of Original ideas and Basic Discoveries in Particle Physics, Erice, July/Aug. 1994, Plenum Press, New York and London, ISBN 0-306-45217-0.

[3] F. Englert and R. Brout, Phys. Rev. Lett. 13 (1964) 321.

[4] P.W. Higgs, Phys. Lett 12 (1964) 132; Phys. Rev. Lett. 13 (1964) 508; Phys. Rev. 145 (1966) 1156.

[5] S.W. Hawking, Commun. Math. Phys. 43 (1975) 199; J.B. Hartle and S.W. Hawking, Phys.Rev.D13 (1976) 2188.

[6] G. 't Hooft, J. Geom. and Phys. 1 (1984) 45.

[7] G. 't Hooft, Nucl. Phys. B256 (1985) 727.

[8] G. 't Hooft, Dimensional Reduction in Quantum Gravity, Essay dedicated to Abdus Salam, Utrecht preprint THU-93/26; gr-qc/9310026; Black holes and the dimensionality of space-time. Proceedings of the Symposium "The Oskar Klein Centenary", 
19-21 Sept. 1994, Stockholm, Sweden. Ed. U. Lindström, World Scientific 1995, 122137.

[9] G. 't Hooft, Class. Quant. Grav. 16 (1999) 3263; also published in: Fundamental Interactions: from symmetries to black holes (Conference held on the occasion of the "Emritat" of Franois Englert, 24-27 March 1999, ed. by J.-M. Frre et al, Univ. Libre de Bruxelles, Belgium, p. 221, gr-qc/9903084.

[10] G. 't Hooft, Determinism in Free Bosons, SPIN-2001/07, ITP-UU-01/14, hep-th 10104080; id., Quantum Mechanics and determinism, in Proceedings of the Eighth Int. Conf. on "Particles, Strings and Cosmology, Univ. of North Carolina, Chapel Hill, Apr. 10-15, 2001, P. Frampton and J. Ng, Eds., Rinton Press, Princeton, pp. 275 - 285; ITP-UU/01/18, SPIN-2001/11, hep-th/0105105.

[11] G. 't Hooft, Determinism beneath Quantum Mechanics, Proceedings Temple University Conf. "Quo vadis Quantum Mechanics?", September 2002, ITP-UU-02/69, SPIN-2002/45. 